Aus dem Hygiene-Institut der Universität in Zürich. (Direktor: Prof. Silberschmidt.)

\section{Ueber die physiologischen Grundlagen der Blutstillung und über ein neues Blutstillungsmittel (Thrombosin).}

Von I. Hirschfeld und R. Klinger.

Die Verhütung größerer Blutverluste bei ausgedehnten oder schwer zugänglichen Gefäßverletzungen gehört nocl heute zu den schwierigeren Aufgaben der Medizin. Trotz mancher auch auf diesem Gebiete gemachten Fortschritte kommt es nicht selten vor, daß eine Blutung erst nach Stunden oder Tagen zum Stehen gebracht wird, sodaß eine beträclitliclıe Schwächung des Kranken, ja selbst Tod durch Verblutung nicht verhindert werden kann. Es dürfte daher von Interesse sein, die Möglichkeiten einer rationellen Blutstillung, welche sich namentlich auf Grund der neuerell Forschungen über das Wesen des Gerinnungsvorganges ergeben, lier kurz zu besprechen.

Die Blutstillung kann durch physikalische (mechanische) oder durch chemische Mittel erreicht werden. $Z u$ den ersteren gelı̈rt die Ligatur, welche in radikaler Weise größere Gefäße verschließt, ferner Kompression und Tam. ponade, wodurch ein vorübergehender Druck auf die blutenden Gefäße ausgeübt und die Blutung solange herabgesetzt wird, bis durch die physiologischerweise einsetzende Gerinnung ein Verschluß der Gefäße eingetreten ist.

Mit dieser Gruppe, speziell mit der vor übergehenden Kompression eines Gefäßes oder Gefäßbezirkes nahe verwandt ist lokale Kälteapplikation, ferner eine Reihe pharmakologischer Blutstillungsmittel, wie Adrenalin, Kokain, No. vokain etc., welche durch Wirkung auf die Muskulatur der betreffenden Gefäße eine Verengerung des Lumens hervorrufen und so die Blutdurchströmung derselben herabsetzen oder zeitweilig unterdrücken. Der Effekt ist somit der gleiche wie bei vorübergehender Kompression, wenn auch anders geartete Kräfte ihn lierbeifülıren. Hier wie dort wird die Blutgerinnung selbst, welche doch immer den endgültigen GefäBverschluß zustandebringen muß, garnicht beeinflußt. Darin äuBert sich die schwache Seite dieser Verfahren; es besteht die Gefahr, daß nach Aufhören des ausgeübtèn Druckes resp. des Gefäßkrampfes die Blutung von neuem einsetzt. 1. Eine andere Art der Blutstillung bewirken Substanzen, welche eiweiBfällende Eigenschaften laben; hierher gehören Salze mancher Sohwermetalle, wie Eisenchlorid, ferner Hitze (Thermokauter). Diese Mittel bringen eine sofortige und massive Koagulation des Blutes zustande, ihre ätzende und nekrotisierende $W$ irkung trifft aber nicht nur die Gefäße, sondern die ganze umliegende Wundfläche. Diese Blutstillung kann daher vom physiologischen Standpunkt aus nicht als eine günstige bezeichnet werdell. Die koagulierten Eiweißkörper wirken wie Fremdkörper, die gebildeten Tlıromben werden schlechter organisiert, lösen sich leicht von den geschädigten Gefäßwandungen und geben zu Nachblutungen Anlaß. Auch dürfte die Widerstandsfähigkeit der die Wunde umgebenden, mehr oder weniger alterierten Gewebe gegenüber Infektionen heratgesetzt cein. Der natürliche Gerinnungsvorgang (speziell die Thrombinbildung) wird dagegen durch solche Mittel nur ungünstig beeinflußt; so läßt sich im Gerinnungsversuch nachweisen, $\operatorname{da} \beta$ z. B. Zusatz von Eisenchlorid schon in sehr kleinen Mengen die Thrombinbildung vollständig verhindert.

Die bisher erwähnten Mittel können nur lokal (am Orte der Blutung selbst) angewendet werden. Sie stehen daher in einem gewissen Gegensatz zu jenen Substanzen, welche eine Erhöhung der Gerinnbarkeit des Gesamtblutes und dadurch eine günstige Beeinflussung von Blutungen bewirken. Bis vor kurzem kannte man von solchen Mitteln bloß die Gelatine, die als $10 \%$ ige Lösung intravenös, meist jedoch nur subkutan verabreicht wird und angeblich gerinnungsbefördernd wirkt. Worauf diese Wirkung der Gelatine beruht, ist noch nicht genügend untersucht, der relativ hohe Kalkgehalt $(0,6 \%)$ dürfte allein wohl kaum eine befriedigende Erklärung geben, da Ca.Ionen in ausrejchender
Menge im Blute zur Verfügung stehen; eher könnte der selır lohe Gehalt an Eiweißabbauprodukten eine Rolle spielen.

In vitro läßt sich übrigens eine Förderung der Gerinnung durch Galatine nicht nachweisen, im Gegenteil zeigten einige von uns geprüfto Gelatinepräparate (darunter das vom Berner Seruminstitut hergestellte, zùr Blutstillung bestimmte sterile Präparat) ( $10 \%$ Lösung), nach Noutralisierung in Mengen von $0,2-0,5 \mathrm{ccm}$ einel $5 \%$ igen Lösung angewendet, nur hemmende Eigenschaften, sowohl gegenüber der Thrombin. bildung wie der Thrombinwirkung. Auch bei Injektion in den Tierkörper ist nach den spärlichen Angaben der diesbezüglichen Literatur sowie nach eigenen Versuchen (intravenöse Injektion von $5 \mathrm{ccm} 10 \%$ iger Galatine bei Kaninehon, Prüfung der Gerinnbarkeit im Paraffinsehälehen vorher, sowie 5 und 45 Minuten nachher) eine Beschleunigung der Gerinllung des Blutes nicht feststellbar.

Nach diesen Ergebnissen der experimentellen Prüfung ist ein hervorragender therapeutischer Effekt von Gelatineinjektionen kaum zw erwarten. Ganz unwahrscheinlich ist ein solcher jedenfalls bei deren Verabreichung per os oder per klisma, falls damit entfernte Blutungen beeinflust werden sollen.

Gegenüber diesen Blutstillungsmitteln scheinen uns diejenigen eine wesentliche Verbescerung $\mathrm{zu}$ bedeuten, welche sich der im Organismus natürlich vorkommenden Faktoren der Blutgerinnung bedienen und durch deren Verstärkung den physiologisclien Gerinnungsvorgang befördern.

Bevor wir auf diese neuen, auf den Ergebnissen der Gerinnungsphysiolog:e beruhenden Methoden der Blutstillung näher eingehen, müssen wir einige Worte über das Wesen des Gerinnungsvorganges vorausschicken. Die Gerinnung des Blutes'erfolgt durch die Ausfällung des Fibrinogens, wodurch unter gleichzeitigem Einschlu 3 der zelligen Elemente die ganze Flüssigkeit erstarrt. Der für das Zustandekommen dieses Vorganges wesentliche Faktor ist ein im Blutplasma vorhandener, wahrscheinlich in die Eeiweilgruppe gehörender Körper, das Serozym (Protlirombin, Thrombogen). Nach dem Austritt des Blutes aus den Gefäßen entsteht aus dem Serozym unter Mitwirkung von Ca-Ionen (wie sie normalerweise in genügender Menge im Blutc vorkommen) das Throm bin (Fibrinferment), welches das Fibrinogen fällt. Die Bildung des Thrombins kann durch gewisse Substanzen (Zytozyme) wesentlich beschleunigt und verstärkt werden. Als derartige, nach Art von Katalysatoren die Thrombinentstehung fördernde Zytozyme können Körper von ganz verschiedener Zusammensetzung funktionieren, wie Lipoide, (Lezithine, Jekorine etc.), Seifen, bestimmte Eiweißabbauprodukte u. a. Ein gut wirksames Zytozym, z. B. eine Emulsion der mit Alkohol aus fast allen tierischen Organen extrahierbaren Lipoide, vermag im Reagenzg]asversuch die Thrombinbildung um das Hundertfache zu steigern und eine entsprechend schnellere Gerinnung einer Fibrinogenlösung herbeizuführen.

Eine rationelle Methode der Blutstillung muB in erster Linie darauf ausgehen, die Thrombinbildung zu verstärken. Wir wissen, daß bei Anwesenheit von reichlichem Thrombin die Gerinnung nicht nur schneller eintritt, sondern auch das gebildete Koagulum be. deutend fester wird.

Eine Steigerung der Thrombinbildung kann am besten durcl Zufulir der an ihr beteiligten Substanzen hervorgerufen werden. Ias Serozym ist hierzu weniger geeignet, denn es ist ein zu labiler Körper, als daß an eine künstliche Zufuhr desselben von außen gedacht werden könnte; hingegen wäre es denkbar, daß $\beta$ Verbindungen gefunden werden - bis jetzt sind solche noch niclit sicher bekannt - welche, durch Injektion oder per os zugeführt, die Serozymbildung im Blute anregen würden.

Ist somit vorläufig eine Verbesserung der Blutgerinnbarkeit durch Erłı̈hung des Serozymgehaltes nicht möglich, so war es um so naheliegender, sterile Zytozymlösungen für diesen $Z$ weck zu versuchen, da die hierher gehörigen Stoffe der praktisclien Anwendung wesentlich geringere Schwierigkeiten bereiten, im tierischen Organismus sehr verbreitet sind und in größerer Monge vorkommen.

Kocher und Fonio gebührt das Verdienst, zuerst diese wichtige Aufgabe in Angriff genommen zu haben. $\mathrm{Zu}$ dem nach ihren Angaben hergestellten Präparat „Koagulen" werden Rinderblutplättchen verwendet, welchen zur Ver- 
mohrung ihrer Wirksamkeit noch Lipoide aus blutbildenden Organen zugesetzt werden. Diese Substanzen sind, mit Traubenzucker vermischt, in Pulverform haltbar und müssen vor Verwendung in siedendem Wasser gelöst werden. Die klinischen Erfahrungen waren nach den Mitteilungen von Fonio günstig').

Die oben erwähnte Tatsache, daß chemisch sehr differente Stoffe Zytozymcharakter aufweisen, stellt uns vor die Wahl, welchen Zytozym wir als praktisch brauchbarem Blutstillungsmittel den Vorzug geben wollen. Für die Praxis sind diejenigen Körper am geeignetsten, welche im gebrauchsfertigen Zustand steril dem Arzte zur Verfügnug gestellt werden können. Ferner ist es wichtig, da $B$ das betreffende $Z$ ytozym neben hoher Wirksamkeit die Eigenschaft der Wasserlöslichkeit besitze. In dieser Beziehung sind die Lipoide als nahezu wasserunlöslich wenig geeignet. Ihre alkoholischen Lösungen könıen nicht verwendet werden, es sei denn stark verdünnt als wäßrige Emulsion, in welcher sie aber nicht haltbar sind. Blutplättchen liefern zwar in der Regel wirksame Zytozynilösungen, scheinen uns aber als Ausgangsnaterial weniger empfehlenswert, weil ihre Gewinnung immerhin einige Schwierigkeiten bietet und die Verwertung eines in relativ sehr geringer Menge im Körper vorkommenden Elementes bei dem großen Reichtum an Zytozym, den der übrige Organismus aufweist, unökonomisch ist.

Im Verlaufe unserer gemeinsan mit Dr. E. Herzfeld unternommenen gerinnungsplyysiologischen Untersuchungen ist es uns gelungen, durch geeignete Vereinigung von Lipoiden mit gerinnungsaktiven Eiweibabbauprodukten Verbindungen zu erzielen, welche sich als sehr wirksames Zytozym erwiese11. Diese Körper bieten zugleich den Vorteil der Wasserlöslichkeit, der Koktostabilität und einer genügenden Haltbarkeit.

Die Prüfung derartıger Präparate auf (eerinnungsahtivitát kann teils $1 \mathrm{~m}$ Reagenzglasversuch, teils $1 \mathrm{~m}$ Tierkorper vorgenommen werden. Für diesbeziigliche Versuche in vitro haben wir, von den Arbeiten von Bordet und Delange ausgehend, ${ }^{2}$ ) ein in grö(Beren Laboratorien leicht ausführbares Verfahren ausgebildet, welches inl wosentlichen darin besteht, daß die zu prufende $Z$ yytozymlosung in steigender Verdiunnung mit Serozym und Ca zusanmengebracht und die hierbei auftrctende Thrombinmenge mit Hilfe von Oxalatplasma gemessen wird. Wir geben in Folgenden ein Beispiel eines derartigen Versuches:

Als Serozym dient Schaf- oder Ziegenserum, das unter bestimmten Vorsichtsmaßregeln aus Oxalatblut gewonnen wurde. (Blut wird aus der Halsvene im Strahle in einem Kölbchen mit Na-Oxalatlosung anfgefangen, wodurch es ungerinnbar wird. Es wird hierauf scharf zentri. fingiert und das erhaltene Oxalatplasma durch Zugabe von $\mathrm{CaCl}_{2}-\mathrm{Lösung}$ zur Gerinnung gebracht. Das Koagulum wird mit einer Zange ausgepreßt (das hierdurch erhaltene Serum ist meist reich an Serozym). In eine Reihe von Reagenzgläschen kommen steigende Verdunnungen des zu prüfenden Zytozyms in je 1,0 physiologischer NaCl-lossung (unverdiinnt, aa, 4, 8, 16, 32 etc.-fache Verdünnung), hierauf je 0,1 Serozym und ein Tropfen einer $1 \%$ igen $\mathrm{Ca}_{2} \mathrm{Cl}$-Lösung. Die Rohrchen bleiben 15 Minuten bei Zimmertemperatur stehen, worauf 1,0 eines verdunnten Oxalatplasinas (nicht rekalzifiert: 4 Teile physiologischer NaCl-Lösung, l Teil 1 \% iger Na-Oxalatlosung, I Teil Oxalatplasma) z.ngesetzt wird. Dieses Plasma gerinnt spontan nicht, dagegen bei Anwesenheit von Thrombin, und zwar um so schneller, je mehr Thrombin zngegen ist. Die Gerinnungszeiten des Plasmas sind in Mintuen in der Tabslle angegeben.

Vergleichsweise wurden im folgenden Versuche einc Lipoidemulsion (alkoholischer Extrakt aus Herzmuskel, 3 \% in NaCl-Lösung enulgiert), cine von uns hergestellte, als "Thrombosin" be zeichnete Zytozymlosung, eine vorschriftsmäßig durch Aufkochen in 10 Teilen Wasser erhaltene „Koagulen"-Lösung Marke ,.Ciba" und eine Oelseifenlösung ( $1 \%$ in Wasser emulgiert) in der angegebenen Weise geprüft.

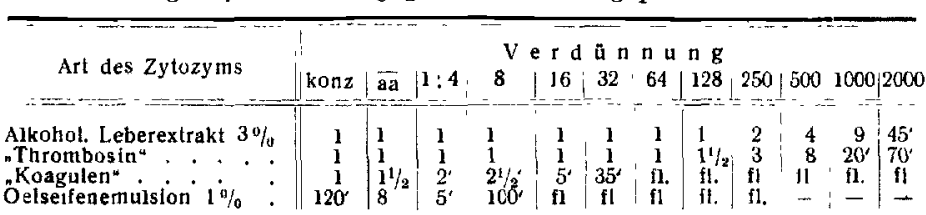

fl. bedeutet Flüssigbleiben des Plasmas noch nach 16 Stunden.

1) Schweiz. Korr Bl. 1913. Grenzgeb. d Med u Chir. 27. 642 Siehe auch Kausch, D. In. W. 1914 Ni. 15. Es scheint, daß das im Handel erhältliche ,Koagulen" nicht stots die gleiche Wirksanıeit aufwcist wie das ursprunglich von Fonio benutzte Präparat; auf eine Umfrage bei einigen Kollegen erhielten wir mehrmals die Antwort, daß sie von der Anwendung dieseg Mittels keinen deutlichen Effek gesehen hatten. - ${ }^{2}$ ) Genauer beschrieben in $\mathrm{D} . \mathrm{m}$. W. $1914 \mathrm{Nr} .32$
Der Versuch zeigt, daß enzelne der geprüften Zytozyme noch in taasendfarher Verdnnnung dic Thrombinbildung soweit verstärkt haheil, daß das Plasma in wenigen Minuten gerinnt.

Die Wirksamkeit im Tierversuch kann entweder in der Weise untersucht werden, $d u B$ frisch aus der Carotis oder aus einer größeren Vene im Strahle entnommenes und in einem paraffinierten ( Blint in der Menge von $1-2 \mathrm{~cm}$ auf paraffinierte Glasschälchen (Uhrglaser) verteilt und das Gerinuungsmittel zugesetz.t wird. Man beobachtet mit Hilfe eines Glasstäbchens, wann die ersten Fibrinfiden auftreten und wann die ganze Bhitınasse erstarrt; oder es wird einem Ticr eine grợßere Menge der Zytozymlosung intravenös inji\%iert und die Gerinnbarkeit des Bhites vol und nach der Injektion ebenfalls in paraffinierten Sohülchen geprüft.

Das folgende Versuchsbeispiel zeigt, daß hierbei eine deutliche Verkırsung der Gerinnungszeit erzielt werden kann, die einige Zeit anhalt :

Kaninchen von $2 \mathrm{~kg}$ Gewicht erhält $4 \mathrm{ccm}$ Thrombosjnlösung intravenös. Bhitentnahme aus der freiprapararierton Carotis vor und nach der Injektion. Cerinnung von je $1-2 \mathrm{ccm}$ im Paraffinschälchen aufgefangenen Blutes:

Vor der Injektion; erste Fibrinfäden nach $40^{4}$; nach 2 Std. nur udvollstăndig geronnen.

$$
3 \text { Min nach der Injektion: Fibrinfäden nach 4, fest nach } 40^{\circ} \text {. }
$$

Nachdem sich die von uns gefundene Zytozymverbindung im Gerinnungsversuch und in vivo als gut wirksam gezeigt und sich durch Injektionsversuch bei Tieren die vollständige Unschädlichkeit derselben erwiesen hatte, haben wir sie auch bei klinischen Fällen von Blutungen versucht. Das Mittel wurde teils bei Operationen (Prof. Henschen), teils bei inneren Blutungen auf der hiesigen Medizinischen Klinik (Prof. Eichhorst), sowie von einigen praktisohen Aerzten angewendet.

Die bisherigen Resultate waren recht befriedigend; nament. lich in Fällen von Hämoptoë bei Lungentuberkulose wurde nach intramuskulärer Injektion wiederholt eine prompte Wirkung beobachtet; mehrmals kamen schwere, seit Wochen bestehende Lungenblutungen nach einmaliger Injektion in kurzer Zeit zum Stillstand. Wir glauben deshalb, die weitere Erprobung und Anwendung des Präparates auch in größeren Kreisen empfehlen zu köınen ${ }^{1}$.

Was gallz allgemein die Indikationen für Zytozymlösungen betrifft, so darf eine erfolgreiche Anwendung vor allem bei langdauernden Blutungen, namentlich wenn solche an einer schwer zugänglichen Stelle des Körpers eingetreten sind, erwartet werden. Traumatische Blutungen aus inneren Orgønen, Lungenblutungen bei Tuberkulose, Magen- und Darmblutungen (Ulcus ventriculi, Typhus) können durch die rasch einsetzende Erlöllung der Gerinnbarkeit des Blutes zum Stehen gebracht werden. Der Chirurg wird zwær in der Regel ohne Heranziehung besonderer Gerinnungsmittel auskommen, weil die meisten bei Operationen auftretenden Blutungen dank den normalen Gerinnungsfaktoren (unterstützt durch Ligaturen und Tamponade) bekämpft werden können. Immerhin dürften sich auch hier Versuche bei Operationen mit besonders starken Blutverlusten empfehlen, nanientlich wenn es sich um schon vorher stark geschwächte Patienten handelt, sowie in Fällen, in denen die Gerinnungsfähigkeit des Blutes herabgesetzt ist, wie bei Hämophilie. Auch in der Geburtshilfe und Gynäkologie werden zweifellos viele Fälle protrahierter Blutungen günstig beeinflußt werden.

Auf Grund der theoretischen Ueberlegungen sowie der experimentellen Ergebnisse, welche wir im Vorhergehenden dargelegt haben, darf von stark gerinnungsaktiven Zytozymlösungen eine gute Wirkung bei sonst scliwer beeinflußbaren Blutungen erwartet werden. Den bisher verwendeten Blutstillungsmitteln sind sie jedenfalls, und zwar prinzipiell, durch die Art ihrer Wirkungsweise überlegen, da sie eine Steigerung der Thrombinbildung selbst herbeiführen. Wie weit sie sich in der Praxis brauchbar orweisen werden, wird die weitere klinische Nachprüfung zeigen.

1) Das Präparat wird unter dem Namen "Thrombosin" hergestellt und als sterile Lósung in $5 \mathrm{~cm}$-Ampullen abgegeben. 